\title{
Seasonal and Secular Periodicities Identified in the Dynamics of US FDA Medical Devices (1976-2020): Portends Intrinsic Industrial Transformation and Independence of Certain Crises
}

\author{
Iraj Daizadeh ${ }^{1}$ (1)
}

Received: 24 July 2021 / Accepted: 13 August 2021 / Published online: 23 August 2021

(c) The Drug Information Association, Inc 2021

\begin{abstract}
The US Food and Drug Administration (FDA) regulates medical devices (MD), which are pre ated on a concoction of economic and policy forces (e.g., supply/demand, crises, patents), under primarily tw ac inistrative circuits: premarketing notifications (PMN) and Approvals (PMAs). This work considers the dynamics of EDA PM And PMAs applications as an proxy metric for the evolution of the MD industry, and specifically seeks to tes the cistence [and, if so, identify the length scale(s)] of economic/business cycles. Beyond summary statistics, the monthly "ay, 16 to December, 2020) number of observed FDA MD Applications are investigated via an assortment of time series chniques (including: discrete wavelet transform, running moving average filter, complete ensemble empirical no ith adaptive noise decomposition, and Seasonal Trend Loess decomposition) to exhaustively seek and find such perio icities. This work finds that from 1976 to 2020 , the dynamics of MD applications are (1) non-normal, non-stat: ary (frac);onal order of integration < 1), non-linear, and strongly persistent (Hurst > 0.5); (2) regular (non-variance), th lat periodicities following seasonal, 1-year (short-term), 5-6 year (Juglar; mid-term), and a single 24-year (Kuznet - me $\mathrm{m}$-t $\mathrm{m}$ ) period (when considering the total number of MD applications); (3) evolving independently of any speci cexogeno factor (such as the COVID-19 crisis); (4) comprised of two inversely opposing processes (PMNs and PMAs) gestis g an intrinsic structural industrial transformation occurring within the MD industry; and, (6) predicted to co inue its ine (as a totality) into the mid-2020s until recovery. Ramifications of these findings are discussed.
\end{abstract}

Keywords Business cycles $\cdot$ Medical d rices $\cdot$ FDA policy $\cdot$ Regulatory science $\cdot$ Economic dynamics

\section{Introduction}

The history of the Uni, Sta ac (US) medical device (MD) industry is one of Mno on-a complicated evolution encompassing a broad t, eryday (e.g., general purpose thermometers) and cialized (e.g., human-embeddable systems) redical products. At some point in time, each registered $T_{L}$, ate $n$ atter how menial from today's vantage poir s an of certain investments. On a company c. ect level, these investments have not only included the mana / supply side variables (such as the various people processes, and systems required of a research and

Iraj Daizadeh

iraj.daizadeh@takeda.com

1 Takeda Pharmaceuticals, 40 Landsdowne St., Cambridge, MA 02139, USA development firm to idealize, actualize, market, and secure economic rents from the sale of a MD product) but also to meet the national policies enforced by one or more national health bodies to ensure the MD's safe intended use [1]. Thus, an important assignment would be to identify and investigate metrics of output that may be used as proxies to track certain aspects of the sector, including its innovativeness and its general health.

Globally, the MD development process requires supervision and registration with a local health agency, which in the US would be the Food and Drug Administration (FDA)'s Center for Devices and Radiological Health (CDRH). ${ }^{1}$ Starting in 1976, CDRH received congressional mandates to ensure the safe and appropriate use of MDs via the Medical Device Amendments to the Federal Food, Drug, and

\footnotetext{
${ }^{1}$ Here, CDRH, FDA, or Agency may be used interchangeably.
} 
Table 1 Some milestones in FDA device regulation since 1970)

\begin{tabular}{|c|c|}
\hline Year & US Drug Regulation \\
\hline 1970 & $\begin{array}{l}\text { Cooper Committee is established, which "recommended that any new legislation be } \\
\text { specifically targeted to devices because devices present different issues than drugs" }\end{array}$ \\
\hline 1976 & Medical Device Amendments to the Federal Food, Drug, and Cosmetic (FD\&C) Act \\
\hline 1990 & Safe Medical Devices Act (SMDA) \\
\hline 1992 & Mammography Quality Standards Act (MQSA) \\
\hline 1997 & FDA Modernization Act (FDAMA) \\
\hline 2002 & Medical Device User Fee and Modernization Act (MDUFMA) \\
\hline 2007 & FDA Amendments Act (FDAAA), MDUFA II \\
\hline 2012 & FDA Safety and Innovation Act (FDASIA), MDUFA III \\
\hline 2016 & 21st Century Cures Act \\
\hline 2017 & FDA Reauthorization Act (FDARA), MDUFA IV \\
\hline
\end{tabular}

Cosmetic (FD\&C) Act, ${ }^{2}$ and subsequent legislation (see Table 1).

In total, the above laws allowed the FDA to develop regulations that offered an opportunity to regulate the industry while simultaneously promoting its development through applying a classification scheme to MDs based on patient risk via the intended use of a given MD. Simplistically, the greater the risk (and thus higher the class), the most stringent the requirements for receiving registration to market a specific MD in the US. ${ }^{3}$ In a great part, this risk-based approm $h$ led to two key regulatory registration paths: the Prem rketing Notification $(\mathrm{PMN}$; otherwise, known as the $51 \mathrm{\Omega}(\mathrm{k})$, d Premarketing Approval (PMA). The PMN pro ss is rel. tively administratively simpler and results in a clea ce [2], while that of the PMA is (typically) more omplex (as may require clinical data) and results in fo mal approval from the Agency. ${ }^{4,5}$ One must keep in mind impertance that both circuits include an applicati from tic sponsor seeking registration: either a PMN or PM 4 As, the application represents the sponsor's tion of the merits of the MD and its potential viabil in t marketplace. In many ways, the act of submittir the vlication for registration is the sponsor's belief 4 all of th, various inputs (investments) have cumulated into innovative product of interest to the marketpla e. This is particularly true if the product attained registratio atus. Sumulatively, therefore, the total number of P and A Applications [and their sum Total MD C. TA. DMNy] over time would be a key piece of evidence

\footnotetext{
2 Pub. L. 94-295 enacted on May 28, 1976.

${ }^{3}$ Section 513(a)(1) of the FD\&C Act [21 U.S.C. § 360c(a)(1)].

${ }^{4}$ Section 515 of ibid.

5 https://www.fda.gov/medical-devices/premarket-submissions/ premarket-approval-pma.
}

(metric) supporting t'ie lution of innovativeness and/or other economies accociated the MD industry.

In this work the metrics of the regulated MD industry are considered: num er of PMN, PMA, and Total MD Applications. It is pothesized that these metrics would be behave si. rly to those of other economic variables, as each MV (ard thus as a collective) is a resultant comite of va ious input-including those of the firm (e.g., peo $_{\mathrm{h}}$, processes, systems), those of the sector (e.g., sup$\mathrm{V} / \mathrm{d}$ mand mechanics), as well as those of national policy a. - enforcement through a regulatory body. Unlike other conomic variables, however, to the author's knowledge, little is known about the dynamics of these metrics and their potential importance to understanding the various factors that may have influenced their evolution (including its forecast). Importantly, in this case of MD development and the metrics selected, these factors include substantive economic activities (e.g., as crises) and/or health policy (e.g., laws) considerations.

Specifically, the focus of this work is on one key characteristic of economic variables (particular those for which sufficient longitudinal data are available) is the appearance of so-called economic or business cycles in the FDA-regulated MD industry. Cycles are generally described in terms of wave mechanics in which a noticeable peak eventually leads to an trough and recycles-where the peak would be considered the pinnacle of economic prosperity (e.g., expansion) of some sort whereas the trough would be a temporally associated misery in productivity (e.g., contraction). From a certain perspective of exogenic strength, the ebb and flow of the variable would correspond to the time-varying strength of forces pressing on the metric. At least four broadly canonical cycles exist (beyond that of seasonal effects), although there has been advancements (see, e.g., [3]) summarized as: 
- Kitchin Short-Term Cycle [4]: 3.5 years in length. Derived as a generalization "supported by a wide range of, annual statistics for Great Britain and the United States, and especially by monthly statistics of clearings, commodity prices, and interest rates for the two countries (page 10)." Kitchin writes that he agrees with a "Mr. Philip Green Wright when he suggests: 'Business and price cycles are due to cyclical recurrences in mass psychology reacting through capitalistic production. The rough periodicity of business cycles suggests the elastic recurrence of human functioning rather than the mathematical precision of cosmic phenomena (page 14)."

- Juglar Mid-Term Cycle [5, 6]: 6-7 years in length with a 1-2-year precipitous drop. Besomi ([5], page 3) captures Juglar's thoughts that-based on banking, population, price of corn, import and exports, rents and public revenue statistics across England, US, Prussia and Hamburg - there was a "a strict correlation ... and that changes go through specific phases, always the same, and are in concordance in the countries where commerce and industry are more development. From this regularity, Juglar inferred that the common premise to all crises lies in the excesses of speculation and in the inconsiderate expansion of industry and trade (ibid, page 4)."

- Kuznets Medium-Term Cycle [7]: 15-25 years ([8] stated 15-20 years; Kuznets specified approximately (but equal to or greater than) 20 years [see Tables 3 and 4 on pares 204 and 205, respectively in Kuznets, 1930 acro US and Europe and various goods and services (inclu. $g$ with caveat trusts)]. Abramovitz [8] nicely mmariz this perspective in trichotomized phases: a rebu drom depression ("growth rate of output yas accelera ig to maximum (page 351)"), steady \& owth ["smoothed growth rate was high enough to keep labo force well employed. It was interrupted 'w short int recessions, but at cyclical peaks the demanu kabor pressed on supply (351/352)]", fo' ed by a depression or stagnation ["actual output vay fell sharply; smoothed output usually declined on at b "grew very slowly (page 352)]."

- Kondratieff I Term C cle ([9]: 50 years [ $\pm 5-7$ years (ibid, page 111), Kondratieff derives 3 cycles each roughl 50 years (more or less) across a series of econometric. rass rance, England, Germany, the US, and t' "who "ld" (ibid, Table 1, page 110). Importantly, the wthor concludes the following proposals: (1) "long 1. res below ... to the same complex dynamic process in whi $n$ the intermediate cycles of the capitalistic economy with their principal phases of upswing and depression run their course (ibid. page 111);" (2) "during the recession of the long waves, agriculture, as a rule, suffers an especially pronounced and long depression (ibid);" (3) "during the recession of the long waves, an especially large number of important discoveries and inventions in the technique of production and communication are made, which, however, are usually applied on a large scale only at the beginning of the next long upswing (ibid);" (4) "at the beginning of the upswing, gold production increases as a rule... (ibid);" (5) "It is during the period of the rise of the long waves, i.e., during the period of high tension in the expansion of economic forces, that, as a rule, the most disastrous and extensive wars and revolutions occur (ibid)."

Here, the key hypothesis that is tested is; assumit. $\mathrm{MMN}$, PMA and the Total MD Applications a prox'v netric associated with the MD industry (and assu ing herefore these variables act as other econc netrics), do latent periodicities exists? If so, what are time engths of such periodicities. The hypothesis tes w r several statistical approaches, based or two ob tives: (1) to understand the intrinsic nature $\& \mathrm{t} \quad 3$ timeseries (viz., descriptive statistics) and, based on this formation, (2) to resolve any identified perir ficit $s$ accordingly. The statistical routines used to describ

- The datu tyde typical distribution statistics (e.g., 1st, 2nd and higher moments), normality, seasonality, linearity, staticharity, long-range dependency, and structural ak.

TI e periodicities include Refined Moving Average Filter (RMAF), Seasonal Trend Loess (STL), wavelet power spectra, and the Complete Ensemble Empirical Mode with Adaptive Noise decomposition (CEEMDAN).

An explanation of each of the algorithms and why they were selected are part of the Materials and Methodologies section. Thereafter, prima facie results are presented. The manuscript closes with an interpretation of the results and key conclusions including limitations of the study and future directions for continued research.

\section{Materials and Methodologies}

While details of the materials (including data acquisition and preparation) and methodologies (including $\mathrm{R}$ programming code) are presented in the accompanying Supplementary Materials as a means to fully replicate and/or extend this analyses, this section summarizes the data sources and its preparation, as well as the rationale and statistical methodologies used in performing the analyses.

\section{Data Sources and Data Preparation}

The data were focused on applications (and not registrations) as the key hypotheses surrounding efficiencies associated 
with the MD industry (and not, e.g., those of the FDA registration process). The US FDA data are considered in this report as the 'authorized system of record;' thus, PMN and PMA data were obtained from the US FDA repository, as there is no known repository containing failed (that is, nonauthorized for sale) MDs.

- PMNs: The data were obtained from the FDA site: https://www.fda.gov/medical-devices/510k-clearances/ downloadable-510k-files on June 30, 2021. The files included PMN7680.ZIP (1976-1980), PMN8185.ZIP (1981-1985), PMN8690.ZIP (1986-1990), PMN9195. ZIP (1991-1995), and PMN96CUR.ZIP (1996-Current).

- Date Range: May, 1976 to Dec, 2020

- Total Number of Records: 158,961

- PMAs: The data were obtained from the FDA site: https://www.fda.gov/medical-devices/device-appro vals-denials-and-clearances/pma-approvals\#pma (under section "PMA/PDP Files for Downloading" on June 30, 2021. The files included pma.zip, "which contains information about the releasable PMAs (ibid)."

- Date Range: Oct., 1960 to Dec., 2020 (Note: The data were truncated to May 1976 to Dec 31, 2020 to allow direct comparison of the earliest PMN recond. A negligible deletion of 178 records.)

- Total Number of Records: 44,831 (44,805 wi truncation)

These data sources were culled for "DAIERECE. ÉD" (Application); that is, the date the appl ation was received by FDA; and imported into Excel, whe in the dates were counted on a monthly scale and hen expored as CommaSeparated Values (CSV) file for ilpu the R programming environment.

In total, 3 variables 5 npr ad the complete dataset: PMN Applications, PMA App tions, and Total MD Applications (that is, the onthly amber of PMNs and PMAs were simply summe -each with 536 values (the sum of all observations withiy a given month from May 1976 to Decembe $>2$ ). ' o summarize, the 3 time series were:

\section{Tim Series \#1: PMN Applications: MDs seeking PMN} (K) registration.

- Tin, Series \#2: PMA Applications: MDs seeking PMA registration.

- Time Series \#3: Total MD Applications: MDs seeking either PMN or PMA registration.

\section{Statistical Analyses}

The general intent of the statistical analyses were two-fold: (1) to understand the intrinsic nature of the 3 time series (viz., descriptive statistics) and, based on this information, (2) to resolve periodicities accordingly. Note: As discussed further below, certain data attributes elucidated from certain tests necessitated further analysis (see Results) specifically around non-stationarity and long-term nemory.

There are many statistical approaches with a navility to characterize a given dataset including decomp it on (viz., reduction to seasonal, trend, and ndom ('stochastic) contributions and inversely reconstru ing the time series (within some sort of accept ble error) t/rough some additive or multiplicative combin. on), structural changes (viz., identification of mean $\mathrm{ful}$ c...nges in certain distribution attributes), da a (e.g., rection denoising and/ or missing data), a id nensicnality reduction (e.g., techniques to reduee or ido fy the variables that would represent key rop ties of the original variable space) and so on. Herc caro orithms selected were a result of: approprioteness $b_{a} d$ on the time series structure (e.g., non-linear $r y$, non-stationarity), accessibility to the algorithm access via the R Project), as well as the nature the signa, to be resolved (periodicity). Thus, an effort has en made to use known methodologies (where posble and cross-validating the results through either using $d_{1}$ erent approaches (ideally with limited theoretical overap) or exploring the parameter space of a given algorithm. As this work is a result of applying known methodologies, all supportive mathematical formulae are deferred via citation. Unless specified otherwise, all methods presented followed standard implementation and default parameters were used (as appropriate) throughout the analyses.

Step 1: Statistical characteristics of the data.

This step simply explores the distribution of the data from a time series perspective, estimating its general characteristics (e.g., moments) as well as outlining its dynamics [e.g., its stationarity and long-range dependency (LRD)]. Either the characteristics of the distribution or properties of the dynamics may alter the calculations, since-for example -a stationary or non-LRD time series may allow for 'simpler' approaches to the analysis, as the moments would be time invariant or individual signals separable, respectively. The analyses followed the following prescription:

Time series loaded and descriptive statistics performed( [10, 11]: R Package: 'fBasics'; [12]: R Package: 'forestmangr'): In this step, the data were read as a time series into the $\mathrm{R}$ program, and descriptive statistics were assessed via the following tests: 
- Normality ([13]: R package: 'foreach'; [14]: R package: 'nortest'): Anderson-Darling (A-D), Cramer-von Mises $(\mathrm{CvM})$, and Lilliefors (Kolmogorov-Smirnov) $(\mathrm{K}-\mathrm{S})$ normality tests

- Seasonality ([15]: R package: 'seastests'): WO, QS, Friedman and Welch tests

- Nonlinearity ([16]: R package: 'nonlinearTseries'): Teraesvirta's and White Neural Network tests, and Keenan, McLeod-Li, Tsay, and Likelihood Ratio tests

- Stationarity ([17]: R package: 'aTSA'): Augmented Dickey-Fuller (ADF), Kwiatkowski-Phillips-SchmidtShin (KPSS), and Phillips-Perron (PP) Unit Root Tests

- LRD: Qu and Multivariate local Whittle Score type (MLWS) tests ([18]: R package: 'LongMemoryTS'), autocorrelation function (ACF) ([10]: R package 'stats'), and Hurst Exponent ([19]: R Project: 'pracma' (hurstexp); [20]: R Project: 'tsfeatures' (hurst)]

- Order of integration ([18]: R package: 'LongMemoryTS']: Geweke-Porter-Hudak (GPH) estimator of fractional difference

Given that 2 tests (viz., MLWS and Qu test) suggested 'spurious' LRD, yet the Hurst Exponent and the existence of non-zero/non-unity (fractional) order of integration existed; thus, statistical estimation of structural breaks was performed using the standard dynamic programming model of Bai and Perron as implemented by ([21-23]: R Projert: 'strucchange'; [24]: R Project: [tseries']). In this appr ach, the definition of structural break is one in which the a some sort of significant change in the para eters or (linear) regression model. The existence of prea would strongly affect the selection of statistical a1gorithms.

Step 2: Statistical determination of beriodicities latent in the data.

Shorter-term Periodicities: Secanal trèru uecomposition via Loess method (STL) ([10]: R Pau 'stats'), Refined Moving Average Filter ( $\mathrm{B}^{\prime}$ F) ([-5]: R Package: 'rmaf'), and the wavelet powe pec rum y.sing a Morlet wavelet under a smoothing soes consuruction ([26]: R Package: 'WaveletComp', re usea 0 investigate the short-term structure of tho tim eries data. For the latter, the average perior versus the verage power for each method was

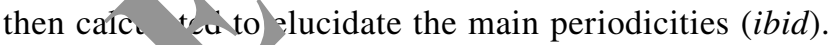
The inan quencies identified were then re-confirmed $x$ spe tral analysis ([27, 28]: R Package: 'forecast'). This app. ch anowed for cross-validation as these methods are orthog nal-that is, there limited-to-no methodological overlap between the methods chosen.

Longer-Term Periodicities: STL, RMAF, and the Complete Ensemble Empirical Mode with Adaptive Noise decomposition (CEEMDAN) ([29, 30]: R Project: 'Rlibeemd') were used to determine the longer trend. The challenge of resolving longer periodicities were multi-factorial and rested with the non-stationary, non-linear, and multiple structural break nature of the data over the duration of the data series. Thus, the CEEMDAN method, which utilizes an adaptive decomposition, has been considered the method of choice to tackle such programs given its flexibility with this type of data [31].

\section{Results:}

\section{Objective 1: Statistical Properties of US MD Applications}

The evolution of PMN and PMA pplications seem to follow inverse trajectories, while th of Tot/1 MD Applications resembles the sum of the o qu. alvely (Fig. 1). The trendline for PMNs (Fi $/ \mathrm{la}) \mathrm{su}$. ests a significant decay since the peak in the $e_{a}$ 1990s, while for PMAs there has been an acceleration sit 2000 (Fig. 1b). While PMA Applications ( $F$ 'g. I ) show a somewhat relative decline in peak in 2020, MD Applications stable due to the clear presence of a single per on th a decline prior to the year of COVID-19 (2020). No : The scales of the trendlines (Fig. 1-right in n) are sl ghtly different than that of the original observation o better resolve the yearly distributions.

$\mathrm{Sh}$ fting our attention to the distribution properties, 1. 1es 2, 3 and Figs. 2 presents the results of the various ests and finds that all three time series are non-normal (skewed with differences in tail thickness: PMN-leptokurtic, PMA-mesokurtic, and total MD- platykurtic relative to a normal distribution, but similar in spread), non-stationary, seasonal, non-linear, with considerable long-memory (see Fig. 2 in which there is a long decay to zero) with fractional order of integration, significant persistency, and the existence of structural changes.

\section{Objective 2: Periodicity Latent within US MD Applications}

Short-term Cyclicity: STL, RMAF, the wavelet power spectrum using a Morlet wavelet, and spectral analysis reconfirmed seasonality as well as elucidated short-term periodicity. Seasonality (Fig. 3) pictographs suggest multiple short-term cyclicity at the 1-year mark or less; spectral analysis resolved dominant peaks at 1-year (PMN), third-year (PMA), and quarter-year (TotalMD), seemingly mapping against business quarters. Where red represents increased foci of energy, the wavelet power spectra (Fig. 4) presents conceptually near similar results, with a 1-year period or less oscillating over the full reporting period for all three time series. Of interest, there is a concentration of energy (red) around 1-year from 1985-1990 for PMNs, 2003-2020 


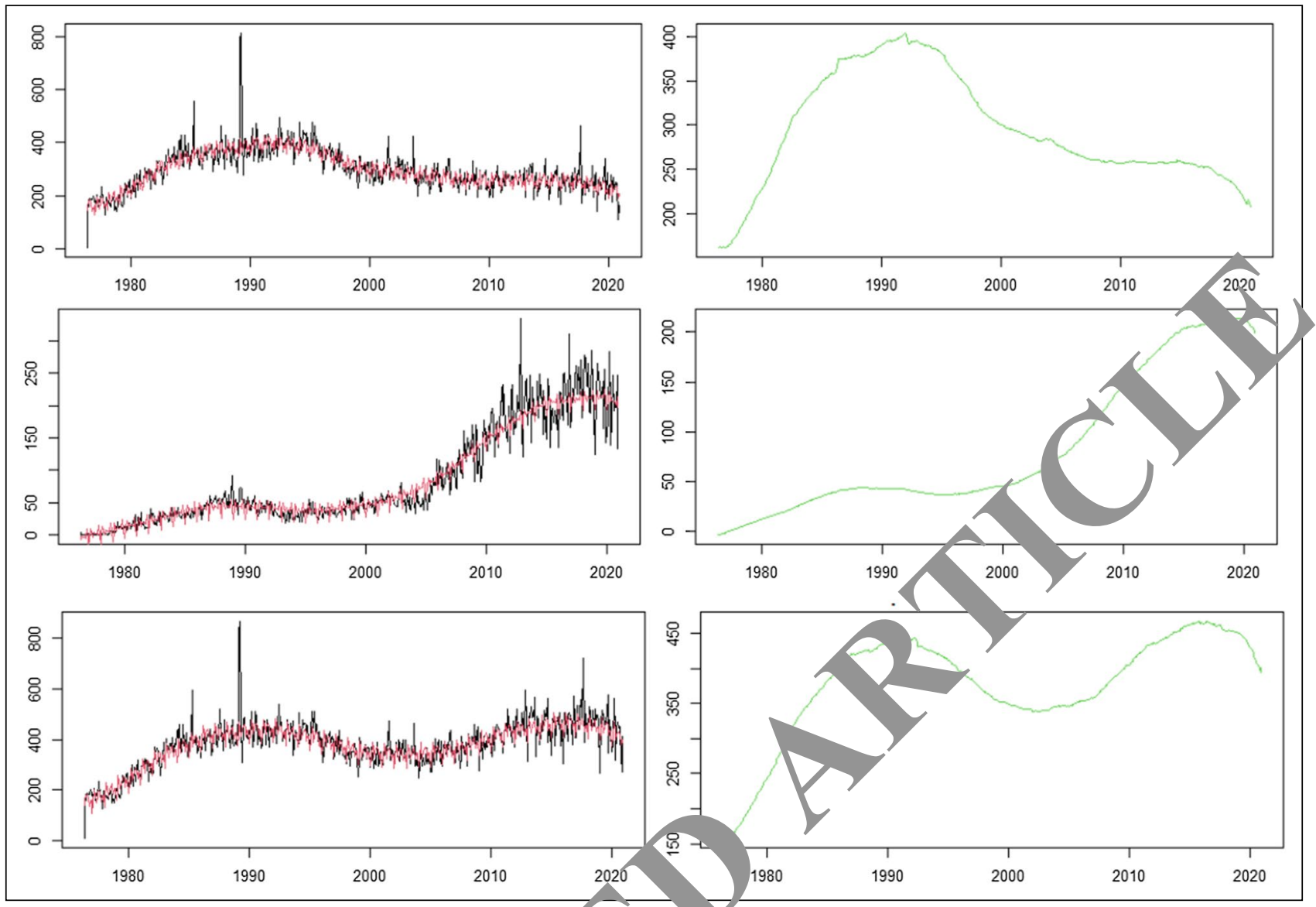

Fig. 1 Time evolution of PMN applications (top), PMA appli ns mid fle), and total MD applications (bottom): observed number of applications (red); estimated trend (left) and estimated trend only (1rght) Fned noving average with a period of 12 months)

Table 2 Summary statistics of US FDA MD applications

\begin{tabular}{llll}
\hline Si & PMN applications & PMA applications & Total MD applications \\
\hline Min mum & 3 & 0 & 7 \\
Ist Quartile & 813 & 335 & 869 \\
3rd Quartile & 247 & 32 & 327 \\
Mean & 347.25 & 135.25 & 437.25 \\
Median & 296.11 & 83.59 & 379.7 \\
Standard error (mean) & 286 & 50 & 388 \\
Lower confidence limit & 3.45 & 3.25 & 4.11 \\
$\quad$ (mean) & 289.33 & 77.21 & 371.62 \\
Upper confidence limit (mean) & 302.89 & & \\
Variance & 6389.99 & 89.98 & 387.78 \\
Standard deviation & 79.94 & 5665.03 & 9058.6 \\
Skewness & 1 & 75.27 & 95.18 \\
Kurtosis & 5.14 & 1.06 & -0.02 \\
Total records & 158,714 & -0.11 & 2.34 \\
\hline
\end{tabular}

Total number of observations $=536$ per time series; rounded to 2 significant digits; units in months 
Table 3 Summary of normality, stationarity, seasonality, long-memory, and non-linearity test results of US FDA MD (rounded to tenths; units in months; rejection of the null hypothesis was based on $p$-value $<0.01$; results are presented in Supplementary Materials)

\begin{tabular}{|c|c|c|c|c|}
\hline \multirow[b]{2}{*}{ Test category } & \multirow[b]{2}{*}{ Tests* } & \multicolumn{3}{|c|}{ Test Result Against Null } \\
\hline & & PMN Applications & PMA Applications & Total MD Applications \\
\hline Normality & A-D, CvM, KS & Reject normality & Reject normality & Reject normality \\
\hline Seasonality & WO, QS, Friedman & Seasonality & Seasonality & Seasonality \\
\hline Linearity & TNN, WNN & Reject linearity + & Reject linearity & Reject linearity \\
\hline Stationarity & ADF, KPSS, PP & Reject stationarity & Reject stationarity & Reject stationari \\
\hline $\begin{array}{l}\text { Order of integration } \\
\text { (fractional differencing } \\
\text { order } d \text { ) }\end{array}$ & GPH & 0.39 & 0.65 & 0.44 \\
\hline Long-memory & $\mathrm{ACF}$ & Yes & Yes & \\
\hline Hurst exponent & $\begin{array}{l}\text { (1) Simple R/S hurst } \\
\text { estimate } \\
\text { (2) } 0.5 \text { plus the maximum } \\
\text { likelihood estimate of the } \\
\text { fractional differencing } \\
\text { order d\# }\end{array}$ & $\begin{array}{l}\text { (1) } 0.83 \\
\text { (2) } 0.93\end{array}$ & $\begin{array}{l}\text { (1) } 0.86 \\
\text { (2) } 1.0\end{array}$ & \\
\hline Structural breaks & $\begin{array}{l}\text { Significance testing of } \\
\text { EFP with OLS-CUSUM, } \\
\text { OLS-MUSUM, Rec- } \\
\text { CUSUM, and Rec- } \\
\text { MOSUM\$ }\end{array}$ & $\begin{array}{l}\text { Reject no structural } \\
\text { changes }\end{array}$ & $\begin{array}{l}\text { Reject no str } \\
\text { chang }\end{array}$ & Reject no structural changes \\
\hline
\end{tabular}

A-D: Anderson-Darling; CvM: Cramer-von Mises; KS: Lilliefors (Kolmogorov-Sn irm PF: Augmented Dickey-Fuller; KPSS: Kwiatkowski-Phillips-Schmidt-Shin; PP: Phillips-Perron MLWS: WO: Webel-Ollech; TNN: reraesvirta Neural Network; WNN: White Neural Network; GPH: Geweke and Porter-Hudak; ACF: autocorrelation function

+ Null hypothesis of linearity (in 'mean') rejected at the $p$-value $<0.0 .8<(\mathrm{TN} \wedge$ nd 0.0087 (WNN)

\#Calculation is difference than that above, see Haslett and Raftery, 1989. nera $y$, the Hurst Exponent is related to the fractional dimension, $d$, by the equation: $d=2$-Hurst

EFP empirical fluctuation processes, OLS-CUSUM:; OLS-MU

for PMAs, and similarly both 1985-1990 a d 2a $2-2020$ for Total MDs.

Longer-Term Cyclicity: RMAF (Fig. ), and CEEMDAN (Fig. 5) algorithms elucidated longer tre 's. Its challenging to view a periodic structure in the $\mathrm{MMAF}$ wrur the exception of Total MD, in which a clear sing e $p$ structure (with two peaks and a trough) solvcd (Fig. 1 bottom). The two respective peaks a loce ad at: April, 1992, July 2016, respectively; a peris of 2 vears and 3 months. The result of the CEEMD methodo ogy depicts peaks at around 5 years across time $h$ ths and time series data.

A sum nary of the periodicities for ease of reference along wit sou ces is presented in Table 4:

\section{Dis 'ssion and Conclusion}

This work concerns itself with the FDA-regulated MD industry and select metrics (PMN, PMA, Total MD) that may be used to explore its evolution. The behavior of the proposed metrics are presumed to be similar to that of other econometrics (e.g., labor, pricing, and production), given the diversity of inputs of varying strengths used to develop
Rer-CUSUM:; Rec-MOSUM:

a specific MD (output). A specific property of econometrics is the presence of periodicities. This work continues to add support for the existence of such periodicities, as several were found via these proposed metrics. The robust finding of periodicities across a broad assortment of econometrics data (including that of FDA-regulated medicines [32]) strongly suggests the existence of potential laws (akin to those identified in physical systems) that may reflect (or indeed govern) aspects of growth and ebb dynamics observed in these curvilinear structures. Future work may consider using these data (and/or those of FDA-approved medicines) to build such a theory, as the data are robust, easy to collect, and relatively granular (daily values that can be aggregated).

Additionally, this work also sought to identify the time lengths of the latent time series periodicities. Importantly, both seasonal and secular cyclicities were identified. These included: seasonal and yearly, mid-term (Juglar), and medium-term (Kuznets) cycles. A longer (Kondratieff) term (>20) year cycle was not observed from the methods used. The seasonal/yearly periodicities as well as a Kuznets 24-year medium--term cycles were the most easily elucidated, based on the selected methods; indeed, the Kuznet 


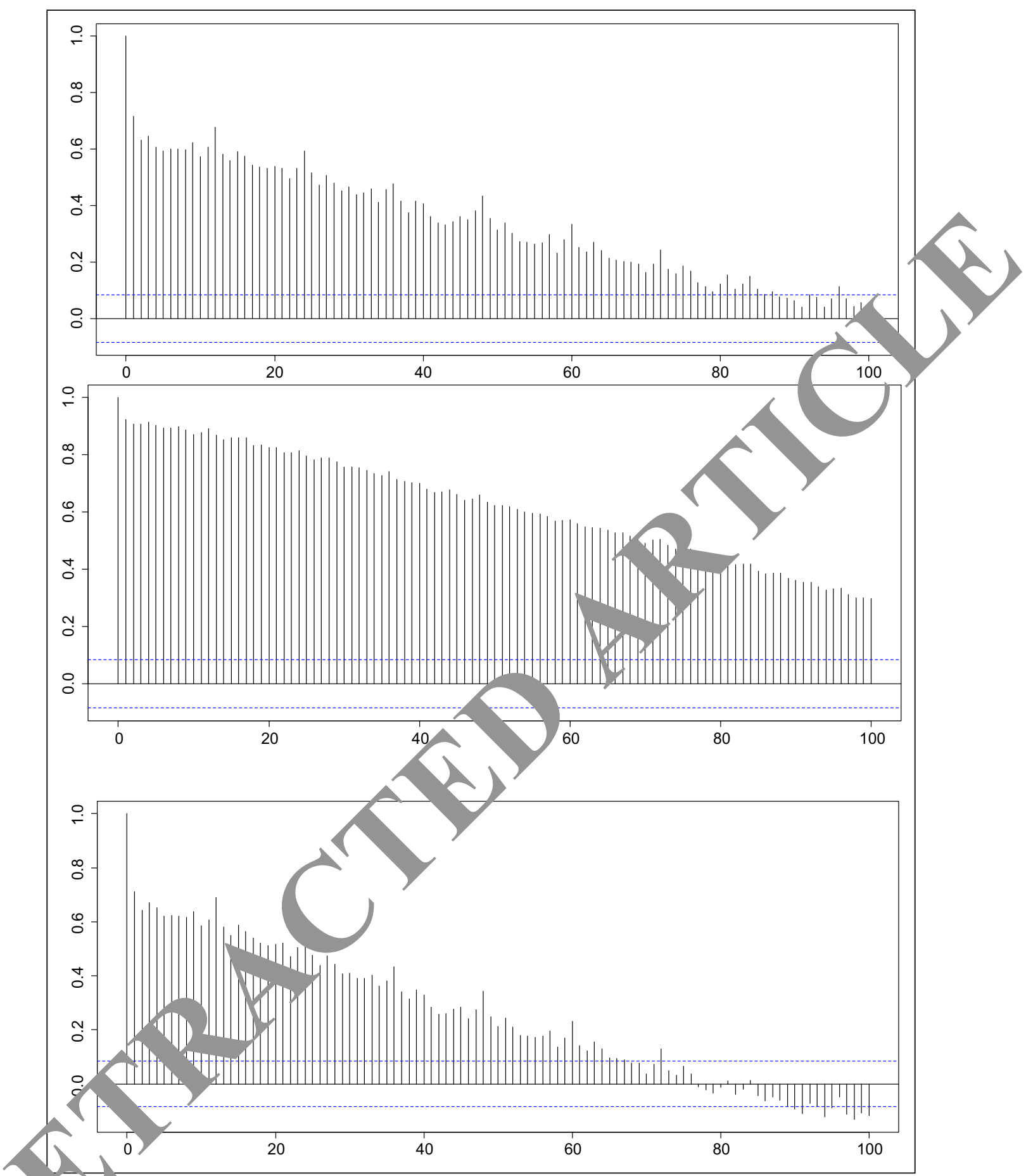

Fig _ to (se correlation function versus lag (months): PMN applications (top), PMA applications (middle), and total MD applications om $05 \%$ Confidence Levels denoted in Blue]

cycle was derived from simple observation (albeit much more clearly post-RMAF).

Theoretically, how would one translate the theorists conjectures of periodicities to the MD industry? For the medium-term (24-year) cycle, and leveraging Kuznets theory, the author speculates that the existence (and use) of the substructures of the PMN and PMA Application curves (metrics) give us a unique insight into the MD industry from a periodicity perspective. Apparently from the simple PMN and PMA plots (Fig. 1), it would seem that the industry is undergoing a potential transformation. The number of PMNs since at least 2000 has been stagnate to trending downward 


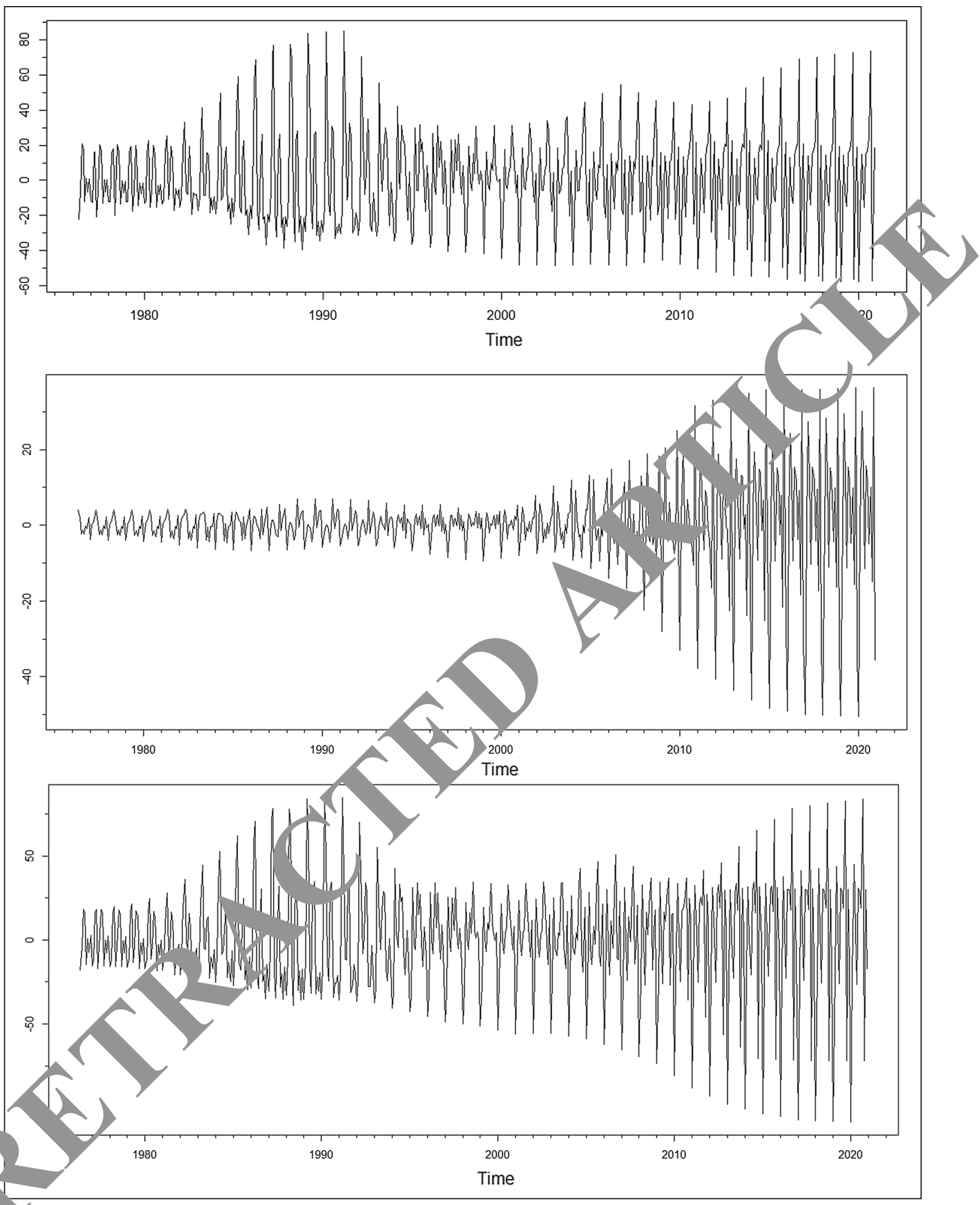

Fig. 3 Seasonal periodicity (via STL) for PMN (top), PMA (mid), and total MD applications (bottom)

from a relative peak in the early 1990s, while PMAs since 2000 has been clearly growing in a striking-cobra-like pattern. Taken together, the collective metric (Total MD) resembles a clear Bactrian-camel-like structure with two clear peaks and a trough in the-1990s and mid-2010s. This would suggest an industry oriented movement from simple 


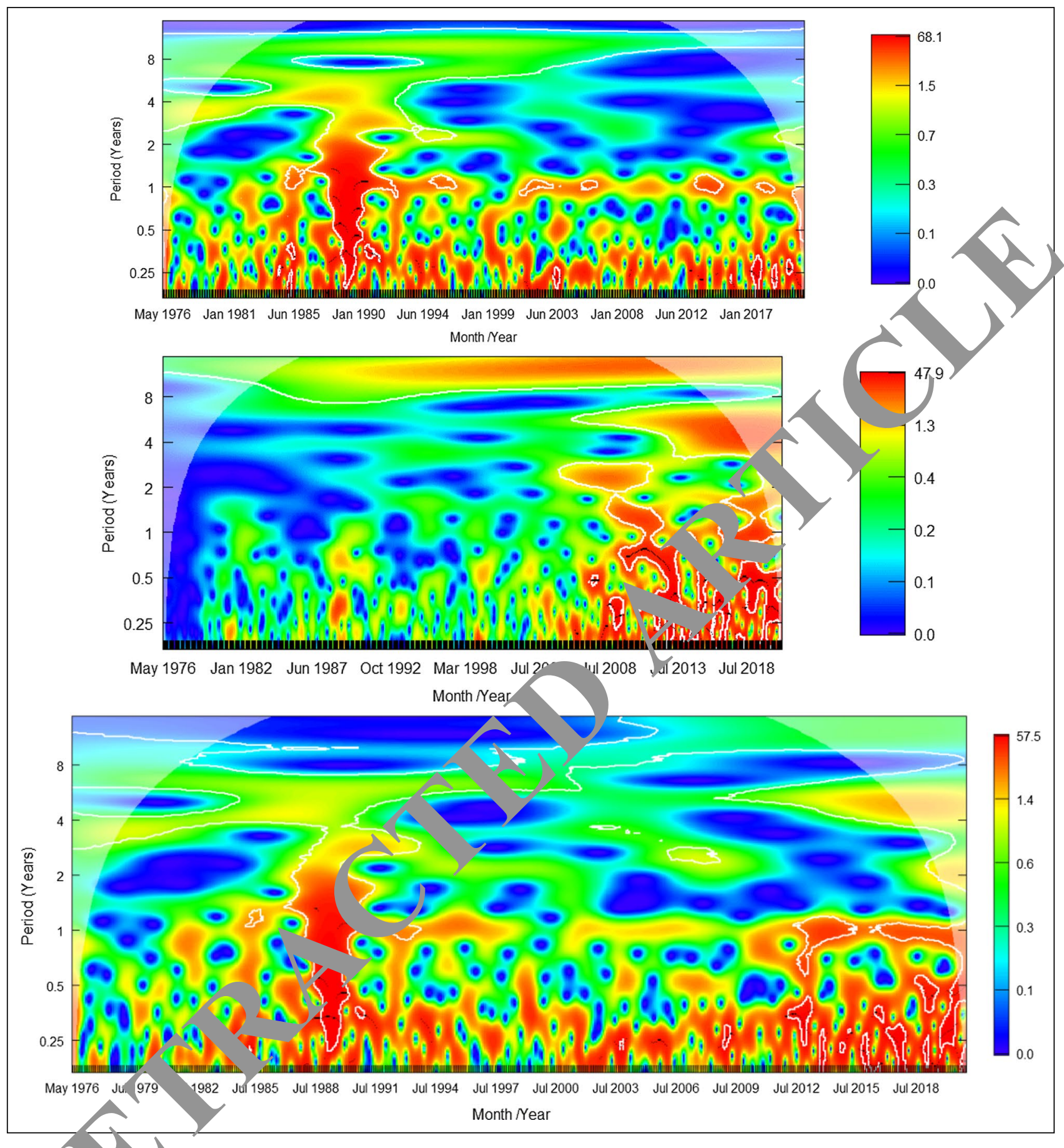

Fig. 4 Waver ower spectra for PMN (top), PMA (middle), and total MD (bottom) applications

(lowe. ik, lower class) MDs to ones that are more complex (higher risk, higher class). Entrepreneurial tendencies grew as of 2000 to build complex MDs (e.g., human embedded systems) requiring additional health authority review and oversight (PMA); presumably driving economic rents given the increase in production costs. PMN activity stagnated due to lack of innovative creativity. Unlike other industries, however, PMAs would not generally replace PMNs-that is, we still need thermometers; thus, there is a floor to PMN Applications, whereas there is no limit to those of PMAs.

The seasonal/1-year and Juglar cycles are also of considerable interest. The latter specifically as the 5-6-year cycle was persistent via both PMAs and PMNs and throughout the data reporting duration of 44 years. An explanation for these 


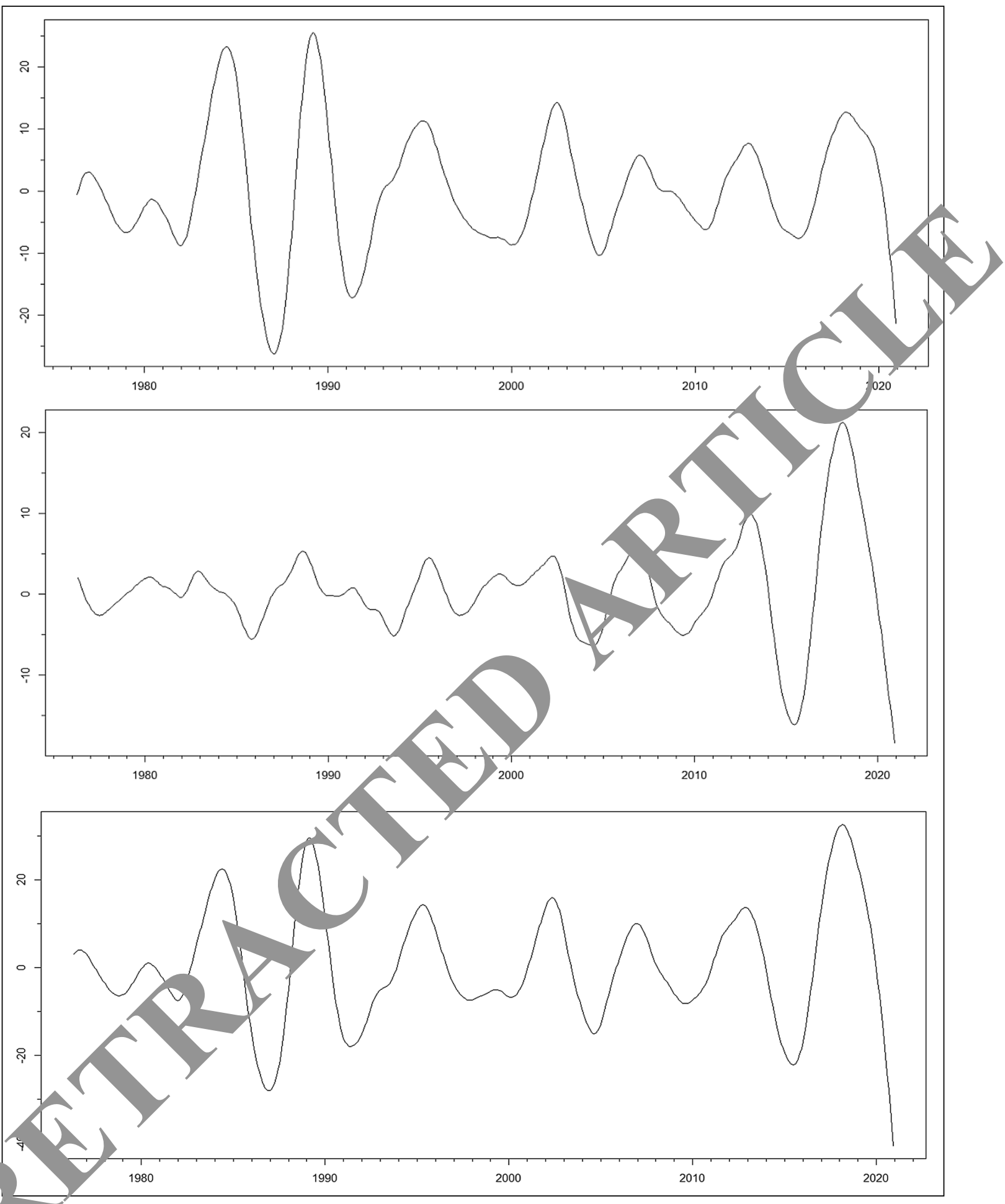

Fig. TEEMDAN trends for PMN (top), PMA (mid), and total MD (bottom) applications

is outstanding but may be speculated to reflect time lengths required for implementing simple to moderate innovation design changes. Imagine a MD in which a specific correction or addition to functionality was made. The updated (new) MD would be subsequently tested, placed into production, an application sought and registration granted by the HA. The rate of MD development in this context would be relatively much shorter than an industry transformation.

As noted by the periodicity theorists, there is little impact of crises to long-term tendencies. Consistent with 

Canonical Economic Cycles with that of periodicities associated with FDA Medical Devices (units in years) as identified in this study (see text for details)
Table 4 Mapping of Broad

\begin{tabular}{|c|c|c|c|c|}
\hline Theory & Canon & PMN & PMA & Total MD \\
\hline $\begin{array}{l}\text { Seasonal/yearly } \\
\text { Cycle* }\end{array}$ & $0.25 / 1$ & 1 & $0.3 / 1$ & $0.25 / 1$ \\
\hline $\begin{array}{l}\text { Kitchin } \\
\text { Short-term cycle }\end{array}$ & 3.5 & & & \\
\hline $\begin{array}{l}\text { Juglar } \\
\text { Mid-term cycle\# }\end{array}$ & $7-11$ & $5-6$ & $5-6 / 8$ & $5-6$ \\
\hline $\begin{array}{l}\text { Kuznets } \\
\text { Medium-term cycle^ }\end{array}$ & $15-25$ & & & \\
\hline $\begin{array}{l}\text { Kondratieff } \\
\text { Long-term cycle }\end{array}$ & $40-60$ & & & \\
\hline
\end{tabular}

the medium-term findings of Kuznets, there is no obvious impact of economic crises on Total MD Applications. There was a subsequent decrease in Total MDs prior to the recent severe acute respiratory syndrome coronavirus 2 (SARSCoV-19; HCoV-19; COVID-19) crisis. In fact, there has not been any noticeable change at least in this study due to the crises on the cycles; the author also did not notice any blatant impact of the COVID-19 crisis on medicines development at least as of Aug 2020 [33]. it would be anticipated from the data that Total MD Application will conti ue a downward ascent until the mid-2020s prior to rising $a_{c}{ }_{1}$, with a potential drop of at least $25 \%$ anticipated $\mathrm{MNs}$ w. continue to drift seemingly. It therefore see is in onable that PMAs would fall, assuming the continued struc

Lastly, this work also has provided isight into the data themselves. We learn that the data ar on-nomal, nonlinear, and non-stationary with sf cific characteristics (lopsided and fat tailed). We also learne ${ }^{2}$. Sere is an intrinsic long-range dependency $(\mathrm{C}-\mathrm{T})$ refiecting memory dynamics as well as multiple uct mal breaks. Both of these features suggest statistical ar wes to generate and investigate hypotheses relata explor $g$ the impact of specific exogenous influences. Th, hronological Hurst Exponent, which algorithm cally leverases LRD, and Structural Breakpoint Analysis $\mathrm{r}$,con $\mathrm{I}$ sed in this way for FDA US medicines as an 2 to 1 . economic events (e.g., crises) and policy i. rve tions to changes in either LRD or structural breaks accu "ingly [34] — a similar experiment could be performed for ML, [35, 36].

This work concludes that (1) PMA and PMN data may be viewed as a proxy measure of innovativeness and certain economies in the MD industry; (2) similar to other econometrics in that periodicities exist are present in these metrics; (3) seasonal/1-year, Juglar and Kuznets periodicities are present in the metrics; (4) these metrics do not seem affected by specific crises (cuch as C D-19); (5) PMNs and PMAs evolve inverse ${ }^{1}$ an suggest a structural industrial transformation; (6) 5 are predicted to continue their decline into the $m_{1}$ 2020s prior to recovery (thus, these metrics $m a$ areater role in predicting the evolution of the MDidustry).

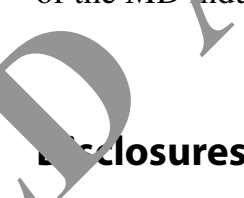

The author is an employee of Takeda Pharmaceuticals; however, this work was completed independently of his employment. As an Associate Editor for Therapeutic Innovation and Regulatory Science, the author was not involved in the review or decision process for this article. See Supplementary Materials for all data and methods to replicate (or extend) the results presented herein.

\section{Acknowledgements}

The author extends gratitude N.D., S.L.D., and N.L.D. for their support of the manuscript.

\section{Supplementary Information}

The online version contains supplementary material available at https:// doi.org/10.1007/s43441-021-00334-4.

\section{References}

1. Gad SC, Spainhour CB, Serota DG. Medical device development. In: Contract Research and Development Organizations-Their History, Selection, and Utilization. Springer, Cham. 2020. https://doi. org/10.1007/978-3-030-43073-3_4 
2. FDA. The 510(k) Program: Evaluating Substantial Equivalence in Premarket Notifications [510(k)]: Guidance for Industry and Food and Drug Administration Staff. 2014. https://www.fda.gov/media/ 82395/download

3. Diebold F, Rudebusch G. 6. Measuring Business Cycles: A Modern Perspective (pp. 117-143). Princeton University Press. 2021. Obtained from https://www.nber.org/system/files/working_papers/ w4643/w4643.pdf

4. Kitchin J. Cycles and trends in economic factors. Rev Econ Stat. 1923;5(1):10-6.

5. Besomi D. Clément Juglar and the transition from crises to business cycle theories. Paper prepared for a conference on the occasion of the centenary of the death of Clément Juglar, Paris, 2 December 2005. Obtained from https://www.unil.ch/files/live/ sites/cwp/files/users/neyguesi/public/D._Besomi_http://citeseerx. ist.psu.edu/viewdoc/download?doi=10.1.1.693.7740\&rep=rep1\& type $=$ pdf

6. Juglar. Des Crises Commerciales et de leur Retour Périodique en France, en Angleterre et aux États-Unis. Paris: Guillaumin. 1862. Obtained from https://gallica.bnf.fr/ark:/12148/bpt6k1060720

7. Kuznets (1930) Secular movement in production and prices: Their nature and their bearing upon cyclical fluctuations. Houghton Mifflin and company, Boston. Obtained from https://dspace.gipe. ac.in/xmlui/handle/10973/24893

8. Abramovitz M. The Passing of Kuznets Cycle. Economica. 1968;35(140):349-67. https://doi.org/10.2307/2552345.

9. Kondratieff ND, Stolper WF. The long waves of economic life. Rev Econ Stat. 1935;17(6):105-15.

10. R Core Team. R: A language and environment for statistical computing. R Foundation for Statistical Computing, Vienna, Austria. 2020. https://www.R-project.org/.

11. Wuertz D, Setz T, Chalabi Y. fBasics: Rmetrics-Markets and Basic Statistics. R package version 3042.89.1. https://CRAN.Rproject.org/package $=$ fBasics 2020

12. Braga SR, de Oliveira MLR, Gorgens EB. forestmangr arèst mensuration and management. $\mathrm{R}$ package version 0.9 .3 . CRAN.R-project.org/package $=$ forestmangr 2021

13. Microsoft; Weston, S. (2020). foreach: Provides For hoopin Construct. R package version 1.5.1. https://CBA.N.R-p ct.org/ package $=$ foreach

14. Gross J, Ligges U. nortest: Tests for Norm lity. R package version 1.0-4. 2015. https://CRAN.R-project.org ckage= ortest

15. Ollech D. seastests: Seasonality Tests. $R$ p. ersion 0.14.2. 2019. https://CRAN.R-project.org $\quad$ age $=$ seastests

16. Garcia CA. nonlinearTseries: Nonl nea ce Series Analysis. R package version $0.2 .11 .2^{\circ}$ https:, CRAN.R-project.org/packa $\mathrm{ge}=$ nonlinearTseries

17. Qiu D. aTSA: Alternat Tes Analysis. R package version 3.1.2. 2015 ittps://C V.R-project.org/package=aTSA

18. Leschinski C. $\mathrm{dMemor}$ is: Long memory time series. $\mathrm{R}$ package versıon 0. 2019. https://CRAN.R-project.org/packa ge $=$ Lor gMemoryTS

19. Borc $\mathrm{H}$ W. Practical Numerical Math Functions. R package

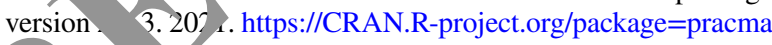
20. Iman vang Y, Montero-Manso P, Talagala T, Wang E, Yar Y O'Hara-Wild M. tsfeatures: Time Series Feature Extracin. N puckage version 1.0.2. 2020. https://CRAN.R-project.org/ p. nge $=$ tsfeatures
21. Zeileis A, Leisch F, Hornik K, Kleiber C. Structural change: an $\mathrm{R}$ package for testing for structural change in linear regression models. J Stat Softw. 2002;7(2):1-38.

22. Zeileis A, Kleiber C, Kraemer W, Hornik K. Testing and dating of structural changes in practice. Comput Stat Data Anal. 2003;44:109-23.

23. Zeileis A. Implementing a class of structural change tests: an econometric computing approach. Comput Stat Data Anal. 2006;50:2987-3008.

24. Trapletti A, Hornik K. tseries: Time Series Analysis and Computational Finance. R package version 0.10-48. 2020

25. Qiu D. Refined Moving Average Filter. R package sio 3.0.1. 2015; https://CRAN.R-project.org/package $=$ rmaf

26. Roesch, A.; Schmidbauer, H (2018). Wave'etComp: Cu utational Wavelet Analysis. R package version https:/CRAN.Rproject.org/package $=$ WaveletComp

27. Hyndman R, Athanasopoulos G, Ber meir C, Cace , G, Chhay L, O'Hara-Wild M, Petropoulos F, Ra ash S, Wing E, Yasmeen F. forecast: Forecasting function for $\mathrm{t}$ serie and linear models. $\mathrm{R}$ package version 8.14. 2021, ast

28. Hyndman RJ, Khand a Y. Auton atic time series forecasting: the forecast package for $\mathrm{K}$ Stat Softw. 2008;26(3):1-22.

29. Helske J, Luuk1 Rlibeem Énsemble empirical mode decomposition (EF TD) a d its complete variant (CEEMDAN). R package version ittps://github.com/helske/Rlibeemd

30. Luukko PJ, Hels Y Räsänen E. Introducing libeemd: a program packa r perforl $4 \mathrm{ng}$ the ensemble empirical mode decomposition. Com at 31(2):545-557. 2016. ISSN 1613-9658, doi: https://c o1.org/10.1007/s00180-015-0603-9

21 Torres M $\%$, Colominas MA, Schlotthauer G, Flandrin P. A comete ensemble empirical mode decomposition with adaptive se. In 2011 IEEE international conference on acoustics, speech a d signal processing (ICASSP) (pp. 4144-4147). IEEE. 2011. Accessible at http://bioingenieria.edu.ar/grupos/ldnlys/metorres/ metorres_files/ICASSP2011_Torres.pdf

32. Daizadeh I. US FDA Drug Approvals are persistent and polycyclic: insights into economic cycles, innovation dynamics, and National Policy. Ther Innov Regul Sci. 2021;55:743-54. https:// doi.org/10.1007/s43441-021-00279-8.

33. Daizadeh I. Has the COVID-19 crisis affected the growth of United States Food and Drug Administration drug approvals? The answer is not yet! A time series (forecasting) study. Ther Innov Regul Sci. 2021;55:553-7. https://doi.org/10.1007/ s43441-020-00249-6.

34. Daizadeh I. Investigating Rates of Food and Drug Administration approvals and guidances in drug development: A structural breakpoint/cointegration timeseries analysis. Ther Innov Regul Sci. 2020;54:1056-67. https://doi.org/10.1007/s43441-020-00123-5.

35. Daizadeh, I. From the Mid-1970s to the Mid-2010s, the Number of US FDA Medical Device Guidelines has been Stagnate: The Key Question is What inspired the Recent Rise? In process.

36. Daizadeh, I. Since the Mid-2010s FDA Drug and Biologic Guidelines have been Growing at a Faster Clip than Prior Years: Is it Time to Analyze Their Effectiveness? Ther Innov Regul Sci. 2021;55:437-439. https://doi.org/10.1007/s43441-020-00233-0. 